\title{
Ex vivo observation of granulocyte activity during thrombus formation
}

\author{
Daria S. Morozova ${ }^{1 \dagger}$, Alexey A. Martyanov ${ }^{2,3 \dagger}$, Sergei I. Obydennyi ${ }^{2,3}$, Julia-Jessica D. Korobkin², \\ Alexey V. Sokolov ${ }^{4}$, Ekaterina V. Shamova ${ }^{5}$, Irina V. Gorudko5 ${ }^{5}$ Anna L. Khoreva², Anna Shcherbina², \\ Mikhail A. Panteleev ${ }^{2,3,6}$ and Anastasia N. Sveshnikova $2,3,6,7^{*}$ (1)
}

\begin{abstract}
Background: The process of thrombus formation is thought to involve interactions between platelets and leukocytes. Leukocyte incorporation into growing thrombi has been well established in vivo, and a number of properties of platelet-leukocyte interactions critical for thrombus formation have been characterized in vitro in thromboinflammatory settings and have clinical relevance. Leukocyte activity can be impaired in distinct hereditary and acquired disorders of immunological nature, among which is Wiskott-Aldrich Syndrome (WAS). However, a more quantitative characterization of leukocyte behavior in thromboinflammatory conditions has been hampered by lack of approaches for its study ex vivo. Here, we aimed to develop an ex vivo model of thromboinflammation, and compared granulocyte behavior of WAS patients and healthy donors.
\end{abstract}

Results: Thrombus formation in anticoagulated whole blood from healthy volunteers and patients was visualized by fluorescent microscopy in parallel-plate flow chambers with fibrillar collagen type I coverslips. Moving granulocytes were observed in hirudinated or sodium citrate-recalcified blood under low wall shear rate conditions $\left(100 \mathrm{~s}^{-1}\right)$. These cells crawled around thrombi in a step-wise manner with an average velocity of $90-120 \mathrm{~nm} / \mathrm{s}$. Pre-incubation of blood with granulocyte priming agents lead to a significant decrease in mean-velocity of the cells and increase in the number of adherent cells. The leukocytes from patients with WAS demonstrated a 1.5-fold lower mean velocity, in line with their impaired actin polymerization. It is noteworthy that in an experimental setting where patients' platelets were replaced with healthy donor's platelets the granulocytes' crawling velocity did not change, thus proving that WASP (WAS protein) deficiency causes disruption of granulocytes' behavior. Thereby, the observed features of granulocytes crawling are consistent with the neutrophil chemotaxis phenomenon. As most of the crawling granulocytes carried procoagulant platelets teared from thrombi, we propose that the role of granulocytes in thrombus formation is that of platelet scavengers.

Conclusions: We have developed an ex vivo experimental model applicable for observation of granulocyte activity in thrombus formation. Using the proposed setting, we observed a reduction of motility of granulocytes of patients with WAS. We suggest that our ex vivo approach should be useful both for basic and for clinical research.

Keywords: Thromboinflammation, Platelets, Granulocytes, Chemotaxis, Flow chambers

*Correspondence: a.sveshnikova@physics.msu.ru

${ }^{\dagger}$ Daria S. Morozova and Alexey A. Martyanov contributed equally to this work.

${ }^{7}$ Department of Normal Physiology, Sechenov First Moscow State Medical University, 8/2 Trubetskaya St., Moscow 119991, Russia Full list of author information is available at the end of the article

\section{Background}

A complex interplay between blood coagulation system, immune system, and endothelium, called thromboinflammation, occurs in diverse pathophysiological situations, such as bacterial infection or cancer [1]. Thromboinflammation is thought to be driven mainly by 
the interactions between granulocytes and platelets [2]. Platelets are non-nuclear cells, produced by the megakaryocytes, that have multiple functions both in hemostasis and immunity [3, 4]. Platelet activation at the site of injury or inflammation leads to the secretion of platelet $\alpha$-granules, which contain P-selectin, fibrinogen, VWF, growth factors, and chemoattractants for leukocytes (NAP2, RANTES, CD40L, etc.) [5, 6]. These proteins play a crucial role in the leukocyte recruitment and adhesion [7-9]. The adhesion to platelets causes leukocytes' integrins activation [10], and their migration through thrombi [11]. Therefore, platelet-leukocyte interactions are in the heart of thromboinflammation.

Granulocytes' migration in thrombi should be dependent on concentrations of their chemoattractants. The contact with a chemoattractant ("priming") of granulocyte leads to their $\beta 2$-integrins (CD11a/CD18, LFA-1 $(\alpha \mathrm{L} \beta 2)$ and mainly CD11b/CD18, Mac-1 ( $\alpha \mathrm{M} \beta 2))$ activation, which results in their firm adhesion to the surface. Therefore, we expect that granulocyte movement in a thrombus should be influenced by their priming. An inhibition of actin polymerization impairs granulocyte chemotaxis [12]. In patients with cytoskeletal abnormalities, for instance, with Wiskott-Aldrich syndrome (genetic hemorrhagic and immunological syndrome; WAS) [13, 14], we expect impaired granulocyte involvement in the thrombus formation process.

The study was aimed at the development and validation of an ex vivo technique, allowing observation and simulation of the thrombus-leukocyte interactions (thrombus growth and leukocyte activity). To validate the method, we loaded the hirudin-anticoagulated whole blood of healthy donors or patients with WAS in parallel-plate flow chambers under the low flow shear rate $\left(100 \mathrm{~s}^{-1}\right)$. The samples from healthy donors were studied under control conditions as well as with leukocyte-priming reagents. We have identified conditions for granulocytes' observation and derived a plethora of parameters for granulocytes' characterization. These parameters were used for the analysis of the whole blood of patients with WAS.

\section{Results}

Granulocytes crawl among the growing thrombi under low wall shear rate conditions

Parallel plate flow chambers are a widely applied modern tool for the hemostasis assessment [15]. We used this tool for the assessment of the leukocyte incorporation into thrombus formation (Additional file 1: Fig. S1; Additional file 2: Video). For this purpose, we used five most known anticoagulants: EDTA and citrate (both chelate calcium), heparin and hirudin (inhibit thrombin indirectly and directly, correspondingly) [16]. For the identification of nuclear cells (NCs), blood was loaded with Hoechst 33342 (intracellular DNA), while DiOC6 (membrane potential) was used to visualize both NCs and other cell types (such as platelets). No adherent NCs were observed in EDTA or citrate anticoagulated blood (Additional file 1: Fig. S1A-H). Upon calcium replenishment to the physiological concentration in the citrated blood (Additional file 1: Fig. S1I-L) as well as in heparin or hirudin anticoagulated blood (Additional file 1: Fig. S1M-T), NCs crawling among the thrombi was observed (Additional file 1: Fig. S1A-C).

We observed rolling or crawling NCs among the thrombi, growing in the flow chamber on fibrillar collagen, as well as some motionless ones (Additional file 3: Video). The cells' instant velocity changed from 0.014 to $0.21 \mu \mathrm{m} / \mathrm{s}$ and the mean velocity was $0.116 \pm 0.017 \mu \mathrm{m} / \mathrm{s}$ (Additional file 1: Fig. S2B, D, F, Table 1). Detailed comparison between the anticoagulant impact on NCs behavior is given in the Table 1 . Although the

Table 1 NC behavior after 30 min of blood perfusion. Data for $N=5$ donors

\begin{tabular}{|c|c|c|c|c|c|}
\hline & EDTA & Citrate & Citrate recalcified & Heparin & Hirudin \\
\hline \multicolumn{6}{|l|}{ NC velocity, $\mu \mathrm{m} / \mathrm{s}$} \\
\hline Minimal instant & $0.072 \pm 0.022$ & $0.07 \pm 0.06$ & $0.022 \pm 0.003$ & $0.018 \pm 0.005$ & $0.022 \pm 0.017$ \\
\hline Maximal instant & $0.65 \pm 0.14$ & $0.58 \pm 0.14$ & $0.62 \pm 0.03$ & $0.567 \pm 0.07$ & $0.703 \pm 0.006$ \\
\hline Average & $0.30 \pm 0.09$ & $0.32 \pm 0.07$ & $0.137 \pm 0.018$ & $0.095 \pm 0.012$ & $0.116 \pm 0.017$ \\
\hline \multicolumn{6}{|l|}{ NC trajectory length, $\mu \mathrm{m}$} \\
\hline Minimal & $30 \pm 21$ & $24 \pm 11$ & $30 \pm 7$ & $23 \pm 9$ & $26 \pm 15$ \\
\hline Maximal & $221 \pm 69$ & $228 \pm 40$ & $361 \pm 114$ & $400 \pm 67$ & $469 \pm 25$ \\
\hline Median & $82 \pm 46$ & $65 \pm 35$ & $148 \pm 62$ & $137 \pm 9$ & $216 \pm 44$ \\
\hline Motionless NCs (velocity < $0.045 \mu \mathrm{m} / \mathrm{s}$ ), \% & - & - & $25 \pm 13$ & $15 \pm 5$ & $7 \pm 4$ \\
\hline Number of attached NCs per FOV & - & - & $0.52 \pm 0.23$ & $1.6 \pm 0.6$ & $1.1 \pm 0.6$ \\
\hline Thrombus area, \% of FOV & $3.6 \pm 2.3$ & $21 \pm 3$ & $19.9 \pm 2.2$ & $9.7 \pm 0.9$ & $12 \pm 4$ \\
\hline
\end{tabular}


citrate recalcification setting allows observation of both plasma and platelet hemostasis, in a large fraction of experiments, the flow chamber becomes occluded by the growing thrombi (data not shown). To avoid it, we added 50 ATU (12.5\% from the standard anticoagulation) of hirudin upon recalcification. Compared to hirudin, in the heparin-anticoagulated blood more NCs attached to the surface (Additional file 1: Fig. S3A), while the NCs trajectories were shorter and the number of motionless NCs was nonsignificantly larger (Additional file 1: Fig. S3B, Table 1). This attests that the NCs are at least primed by heparin, in line with previous data $[17,18]$. Thus, hirudin was used as anticoagulant in all further experiments.

In order to identify the NCs, DiOC6 (Fig. 1D), the antibody to CD66ace (Fig. 1E) and the antibody against CD66ace (Fig. 1F) were used. Alternatively, blood was pre-incubated with Hoechst $33342(\mathrm{H})$, DiOC6 (I), or anti-CD2 antibody (J). It appeared that while most of the cells were granulocytes (CD66b and CD66ace positive; Fig. 1D-G, Additional file 1: Fig. S4A-J)), a subset of cells was T lymphocytes (CD66ace, CD66b negative and CD2 positive; Fig. 1H-K, Additional file 1: Fig. S4KO; Additional file 4: Video). Most of the crawling cells

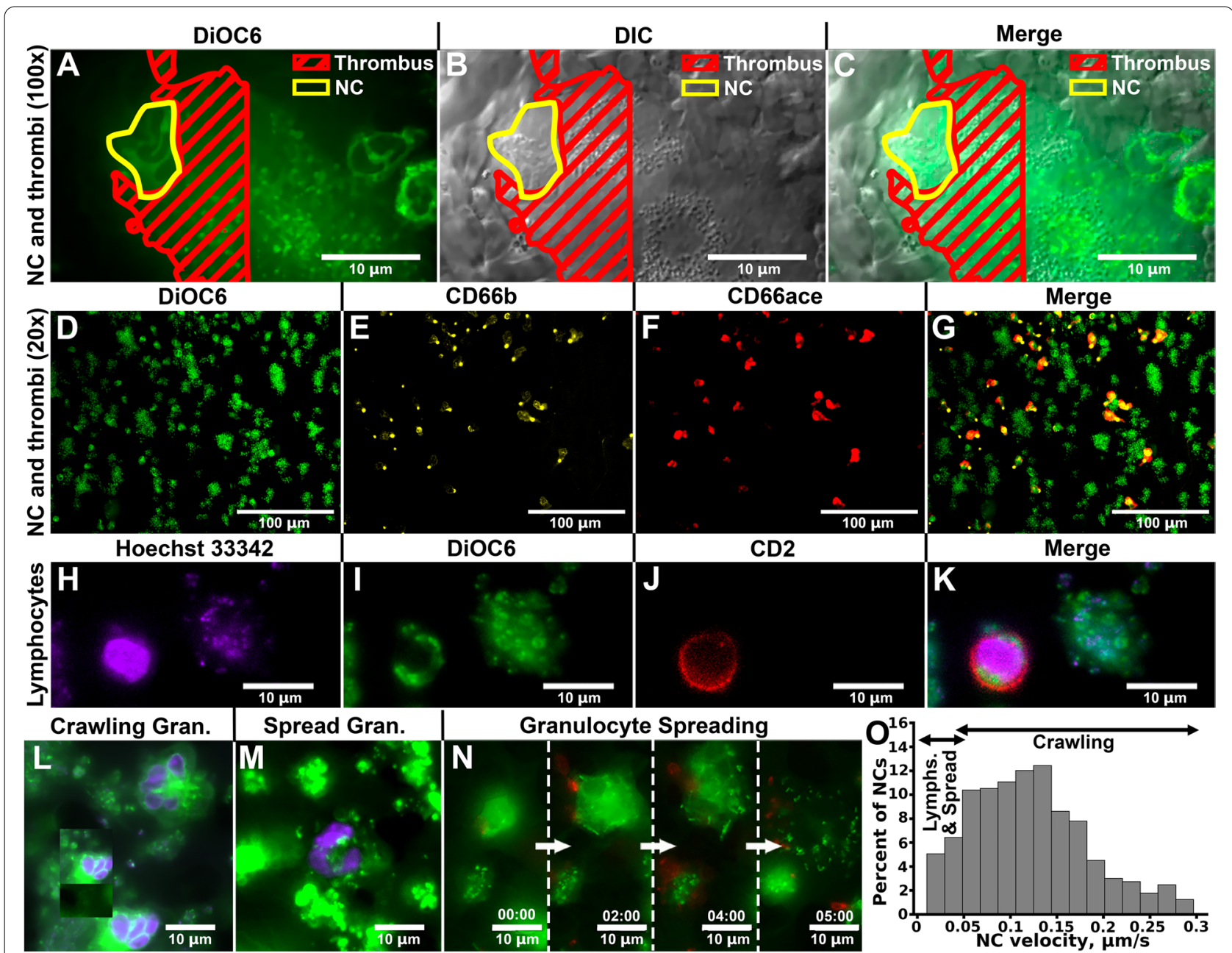

Fig. 1 Nuclear cells (NCs) are able to crawl among the growing thrombi. A-C Thrombi (highlighted by red) with crawling NCs (highlighted by yellow) upon hirudin-anticoagulated blood perfusion through the flow chamber with fibrillar collagen fluorescent mode (DiOC6, A) or DIC (B, C) at $\times 100$ magnification (raw data at https://doi.org/10.6084/m9.figshare.18356042.v1). D-G Thrombi with crawling NCs in the presence of DiOC6 (D), CD66b (E), and CD66ace (F) at $\times 20$ magnification (G-merged D-F; raw data at https://doi.org/10.6084/m9.figshare.18417194.v1). H-K Among crawling CD66b and CD66ace positive cells were motionless cells with single nuclei $(\mathbf{H})$ and clustered DiOC6 staining (I), which appeared to be CD2-positive (T lymphocyte marker; J) as well (K-merged $\mathbf{H}-\mathbf{J}$; raw data at https://doi.org/10.6084/m9.figshare.18435551.v1). L, M Different types of granulocytes were observed_crawling (L) and spread (M). $\mathbf{O}$ Granulocyte slowing down and spreading dynamics. $\mathbf{O}$ Based on the granulocyte and lymphocyte velocity distribution, it can be claimed that spread granulocyte and lymphocytes were distinctive not only by their appearance but by their velocity as well. Representative data out of $N=10$ donors. Individual data values are given in the Additional Table 3 
(Fig. 1L, Additional file 5: Video) appeared to have active CD11b in their posterior part (Additional file 1: Fig. S4AE). Some of the granulocytes slowed down and spread (Fig. 1M) with fewer number of active CD11b in the middle parts of the cell (Additional file 1: Fig. S4F-J) [17]. The process of granulocyte slowing down and spreading is depicted in Fig. $1 \mathrm{~N}$ and in Additional file 6: Video. Both granulocytes and $\mathrm{T}$ lymphocytes were CD18 positive; however, CD18 staining on T lymphocytes appeared to be less clustered (Additional file 1: Fig. S5). The named three cell types (crawling granulocytes, spread granulocytes and $\mathrm{T}$ lymphocytes) were clearly distinguishable by their DiOC6 staining pattern (Fig. 1A, I, L, M) and their velocities, namely, spread granulocytes' and $\mathrm{T}$ lymphocytes' velocity was less than $0.045 \mu \mathrm{m} / \mathrm{s}$ on average (Fig. 1O). The number of all types of NCs increased from 1 to 2 cells per field of view (FOV) on the 5 th minute to 8 cells per FOV on the 20th minute and then did not change significantly up to 30th minute (Additional file 1: Fig. S6). Analysis of the blood contents prior and post blood perfusion revealed that nearly $50 \%$ of granulocytes participated in the process of thrombus formation in our conditions (Additional file 1: Fig. S7A-C). However, upon platelet depletion, slightly yet significantly lesser amounts of granulocytes were incorporated in the thrombus formation process in contrast to non-thrombocytopenic blood (Additional file 1: Fig. S7D-F). On the other hand, granulocyte crawling velocity appeared to be independent from the platelet count: no statistical differences were present between granulocyte crawling in normal and thrombocytopenic blood (Additional file 1: Fig. S8).

\section{Mediators of inflammation and platelet activators alter granulocyte behavior}

In order to test whether NCs activation affected their adhesion at sites of growing thrombi, we performed experiments in the presence of leukocyte-priming agent, myeloperoxidase (MPO) [19], which is one of the key proteins, stored in granulocytes and secreted upon activation [20]. By binding to Mac-1, MPO can induce granulocyte activation in an autocrine fashion including MAPK activation, degranulation [21, 22], and adhesion [23]. It has been demonstrated previously that MPO facilitates granulocyte recruitment by its positive surface charge [24]. Furthermore, MPO-dependent granulocyte recruitment at sites of inflammation has been demonstrated both in vitro and in vivo [24]. MPO did not significantly affect granulocyte trajectories (Fig. 2D-F) and velocities (Fig. 2H), while significantly increasing the number of NCs per FOV (Fig. 2G) and percentage of motionless NCs (Fig. 2I) in line with the literature data on MPO impact on granulocytes [19, $22,24]$. For the combined activation of granulocytes on platelets, we used fucoidan, capable of inducing platelet degranulation (P-Selectin exposure, Additional file 1: Fig. S9) via CLEC-2 receptor [25] as well as pro-inflammatory cytokine production and apoptosis delay in granulocytes [26]. Therefore, we expect an increased attraction of granulocytes to the growing thrombi. Indeed, the number of NCs (Fig. 2G) as well as the NC crawling velocities (Fig. $2 \mathrm{H}$ ) increased upon fucoidan treatment, while $\mathrm{NC}$ spreading was not significantly altered (Fig. 2I). Finally, we used lipopolysaccharides (LPS) [27] to mimic the proinflammatory granulocytes stimulation. LPS activate and promote NETosis via TLR4 receptor on granulocytes, while do not significantly alter platelet functioning and thrombus formation in flow chambers [28]. As expected, LPS increased granulocyte crawling velocity only at 10th minute but significantly reduced it at 20th and 30th minute (Fig. $2 \mathrm{H}$ ), which was associated with the statistically significant increase of the numbers of slow NCs upon LPS introduction (Fig. 2I). All of the used agents altered the thrombus area; however, their effects are not uniform and should be the object of additional studies (Additional file 1: Fig. S10, S11A).

\section{NCs bear Annexin-V-positive platelets}

Among the key physiological functions of granulocytes in the blood flow is the removal of the phosphatidylserine (PS) exposing (Annexin-V-positive) cells [29]. In particular, granulocytes can form hetero-aggregates with Annexin-V-positive platelets [7], which form in the process of thrombus formation [30]. Indeed, in our experimental setting, the crawling granulocytes were associated with Annexin-V-positive particles (Fig. 3AC). In order to identify these particles, whole blood was loaded with Hoechst 33342, anti-CD61 (specific marker of platelets) antibodies, and Annexin-V. It appeared, that the majority of granulocytes were associated with Annexin-V-positive and CD61-positive cells-procoagulant platelets (Fig. Additional file 1: 2A-E, Additional file 7: Video). Same has been observed for experiments at lower magnification $(\times 40)$ : most of granulocytes appeared to be bearing Annexin-V- and CD61-positive cells (Additional file 1: Fig. S12F-I). To identify the mechanism of Annexin- $\mathrm{V}$-positive platelets association with crawling NCs, we analyzed active CD11b and CD66b distribution on the crawling cells (Fig. 3 and Additional file 1: Fig. S13, correspondingly). Colocalization analysis [31] revealed that DiOC-6 (Fig. 3G) and CD66b (Additional file 1: Fig. S11D) staining did not significantly correlate (Pearson's correlation coefficient, PCC, $0.46 \pm 0.13$ and $0.52 \pm 0.15$, correspondingly) with Annexin-V fluorescence (Fig. 3I). On the other hand, the correlation between CD11b and Annexin-V fluorescence (Fig. 3H) was significantly higher, $\mathrm{PCC}=0.68 \pm 0.12$ (Fig. 3I). Therefore, platelets attach to crawling granulocytes, 


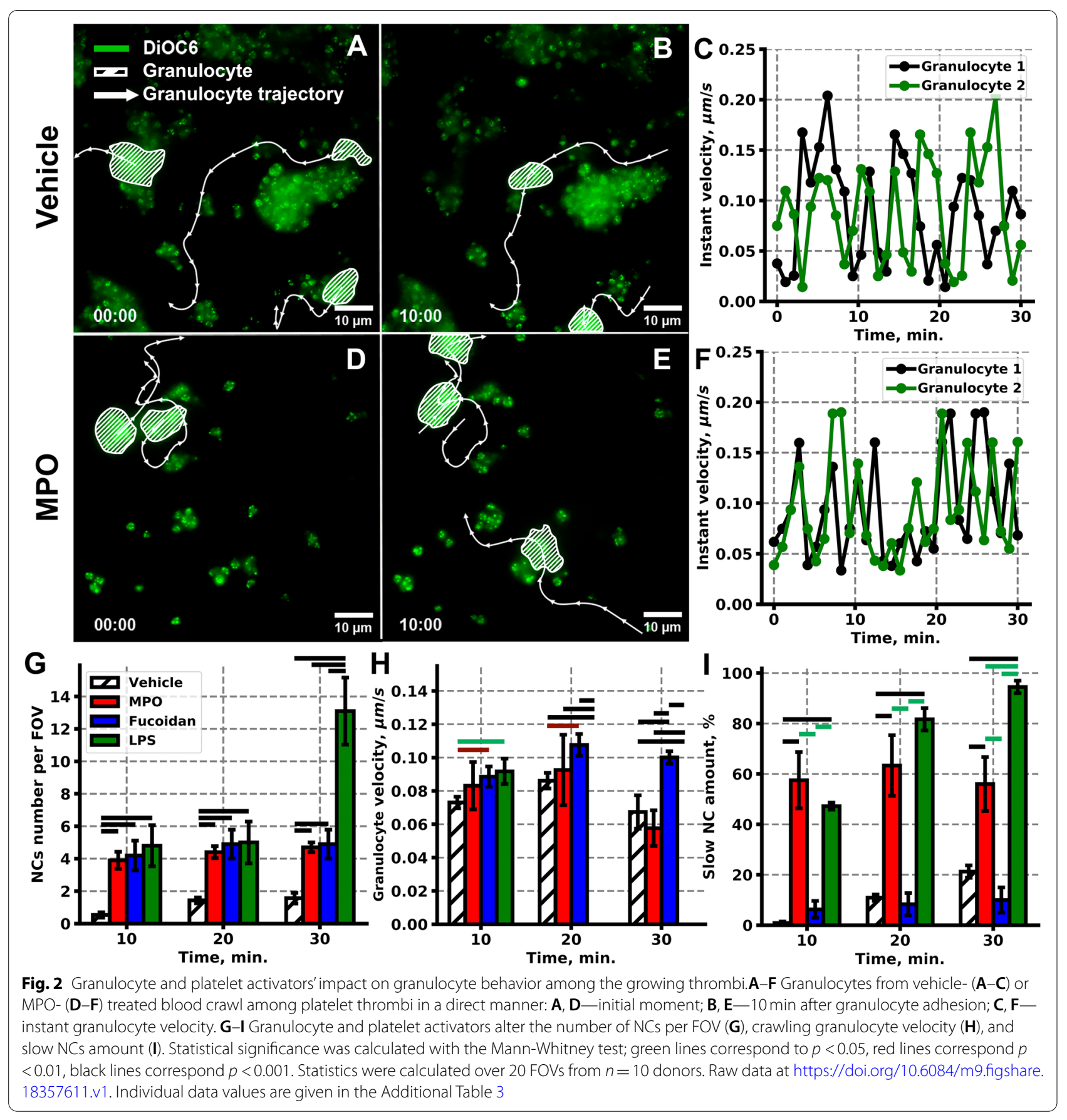

probably, in a CD11b-dependent manner. Furthermore, trajectories of the crawling NCs correlated to the trajectories of Annexin-V-positive platelets in most cases (Fig. 3L, Additional file 7: Video).

\section{Blood plasma proteins are required for granulocyte crawling among the thrombi}

In order to identify the mechanisms of the NC incorporation at sites of growing thrombi, we have removed plasma proteins from the whole blood by means of sequential centrifugation (see the "Methods" section). In a such "clear" system, no granulocyte crawling on the collagen surface was observed (Fig. 4A), while short-term $\mathrm{NC}$ attachment to the platelet covered surface and granulocyte rolling was observed $6 \mu \mathrm{m}$ above the collagen layer (Fig. 4B, Additional file 8: Video). The introduction of $10 \%$ of physiological concentrations of fibrinogen and von Willebrand factor (VWF) resulted in the recovery 


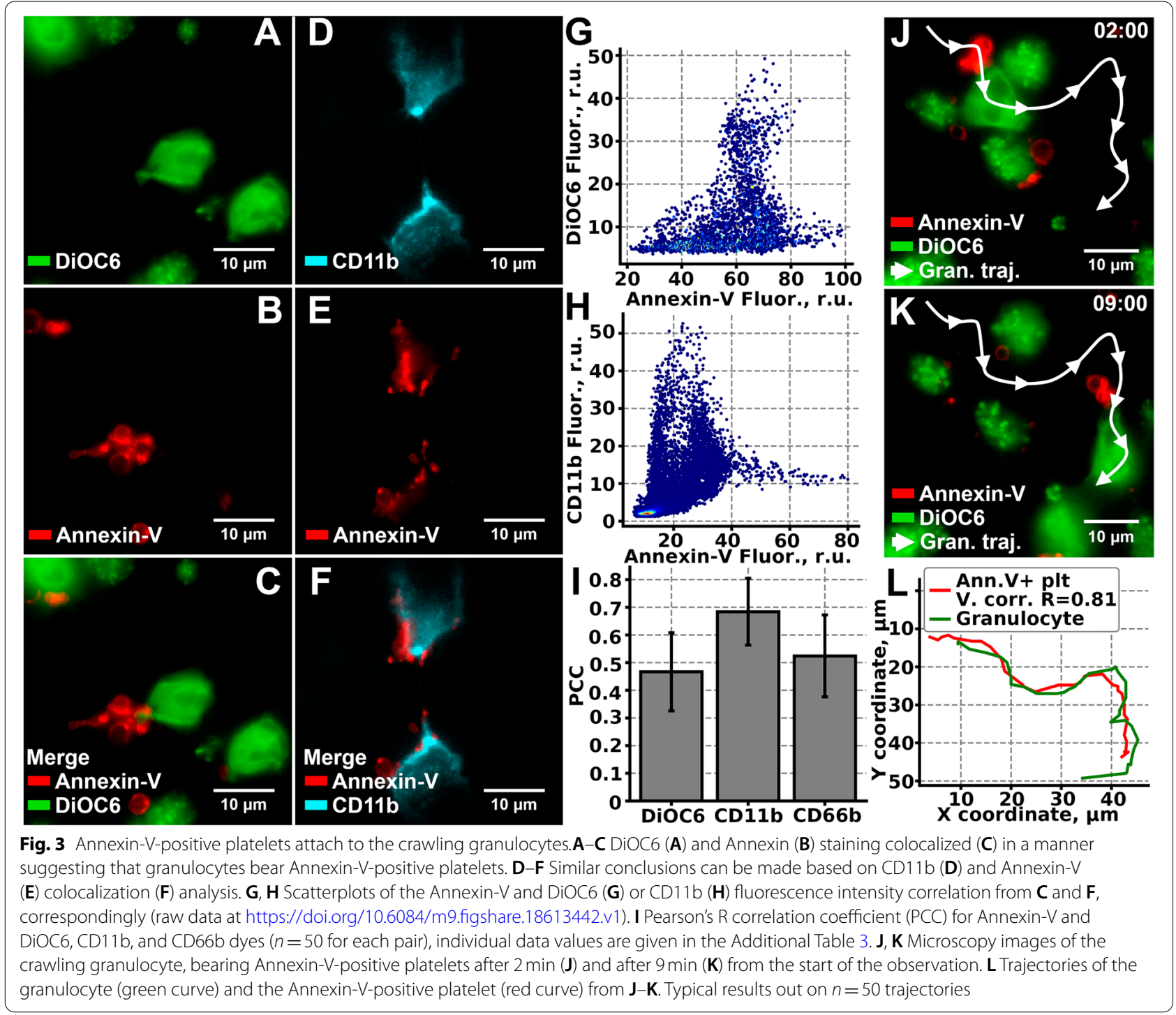

of granulocyte crawling (Fig. 4C, D, Additional file 8: Video) on the collagen covered glass, yet granulocytes descended from the thrombi less readily than in the whole blood (Fig. 4E, F, Additional file 8: Video). Based on these findings, we propose the following scheme of the events: NCs from the blood flow attach to the growing thrombi in the presence of calcium and descend onto the collagen level via fibrinogen and VWF, where granulocytes crawl, bearing Annexin-V-positive platelets and eventually slow down and spread (Fig. 4G).

\section{Crawling of WAS patients' granulocytes is altered in comparison to healthy donors}

Altogether, the observed features of granulocytes crawling are consistent with the granulocyte chemotaxis phenomenon. Therefore, we assumed it should be altered in cells with defective cytoskeleton. Wiskott-Aldrich syndrome is a genetic disease, caused by WAS gene mutations and alteration of cytoskeleton of both immune cells and platelets [32]. WAS is mainly characterized by immunodeficiency, microthrombocytopenia, and autoimmune/oncological predisposition [14].

Typical FOVs of healthy donors and WAS patients are shown in Fig. 5A-C and D-F, correspondingly. The number of granulocytes per FOV was increased (Additional file 1: Fig. S11C) in WAS patients in comparison to healthy donors. This resulted in a significantly increased ratio of granulocyte number to thrombus area in WAS samples (Fig. 5G, Additional file 1: Fig. S11B). On the other hand, crawling granulocytes of WAS patients were significantly slower than crawling granulocytes of healthy donors (Fig. 5G). Finally, slow NC amount was significantly increased in WAS samples in comparison to healthy donor samples (Fig. 5H). Thus, it can be claimed 


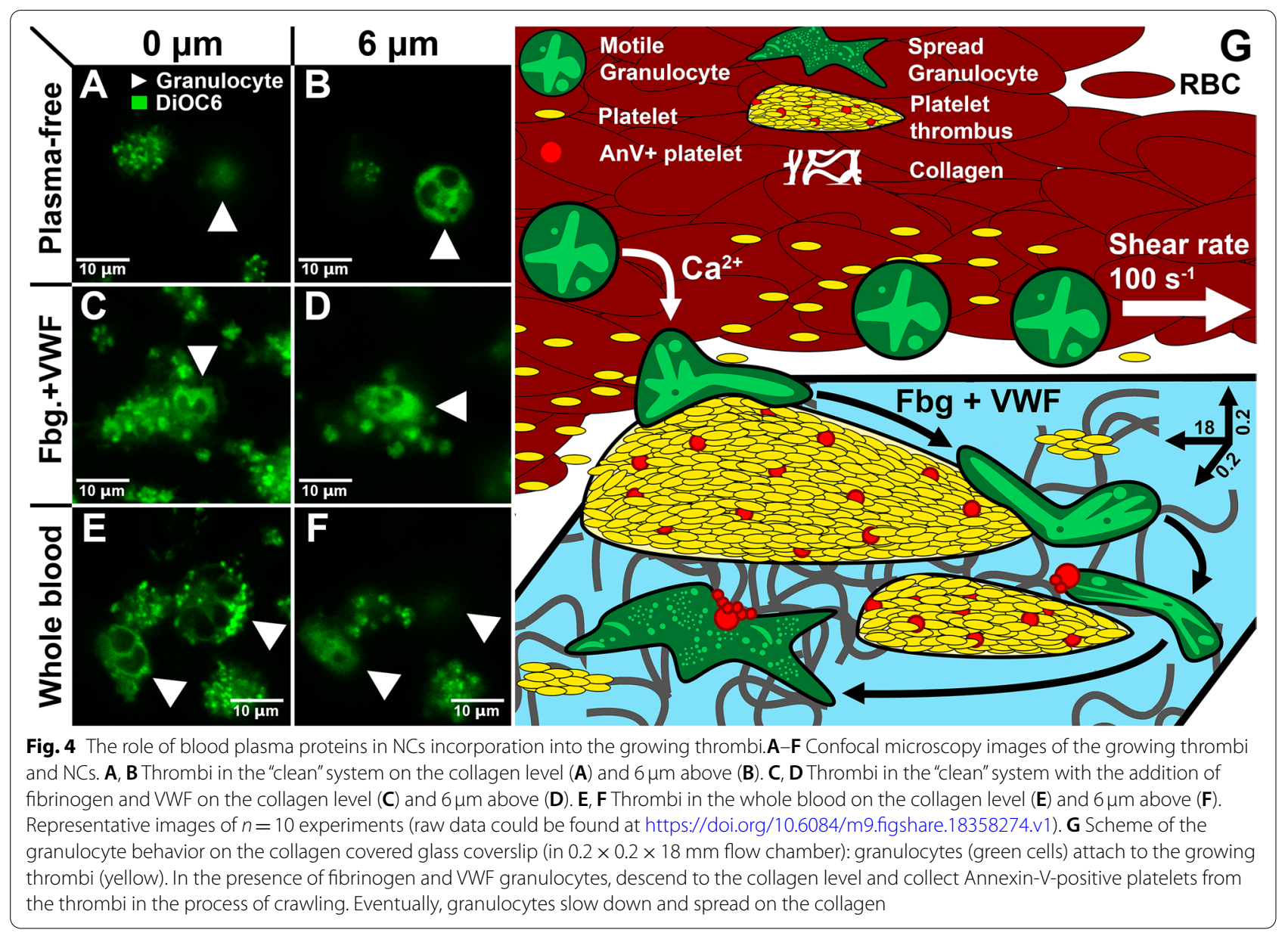

WAS granulocytes and MNCs are more prone to the collagen adhesion and spreading. In order to check, whether granulocyte velocity are not altered due to thrombocytopenia or neutropenia, we partially supplemented leukocyte rich plasma with Tyrode's, analogous to our earlier study [33]. It appeared, that in the "neutropenic" conditions no alterations in the granulocyte crawling were present (Additional file 1: Fig. S11D).

Finally, experiments with "hybrid" samples were performed. Blood samples from patients with WAS were platelet-depleted and substituted with healthy donor's platelets. Washed healthy donor platelets were loaded with Fura-Red in order to distinguish them from the native WAS patient cells (Additional file 1: Fig. S14 A-O). It appeared that the observed thrombi were Fura-Red positive, which allowed us to claim that platelet replacement was effective. Alternatively, effectiveness of the replacement was controlled using a cell counter: platelet count in the whole blood dropped upon depletion and then increased upon healthy donor platelet addition (Additional file 1: Fig. S14P). Furthermore, platelet size also increased upon replacement, what additionally confirms validity of the developed experimental setting, as WAS patients typically have smaller platelets (Additional file 1: Fig. S14R). Granulocyte numbers in patients remained intact (Additional file 1: Fig. S13S). Average velocities of the granulocytes of WAS patients in the presence of WAS platelets and healthy donor platelets did not differ significantly (Additional file 1: Fig. S14T), while the amount of spread granulocytes and lymphocytes even increased (Additional file 1: Fig. S14U). As granulocytes of the WAS patients in were generally slower than granulocytes of healthy donors (Fig. 5G), it can be stated that substitution of the defective platelets of the patients by healthy donor platelets does not alter WAS patient granulocyte crawling.

\section{Discussion}

Here, we developed an ex vivo approach to study granulocyte involvement into the thrombus formation. We observed leukocytes rolling, crawling, and arrest in thrombus formation (Fig. 1). The granulocytes behavior was affected by MPO, fucoidan, and LPS (Fig. 2). Most of granulocytes were bearing procoagulant platelets, presumably, in CD11b-dependent manner (Fig. 3). 


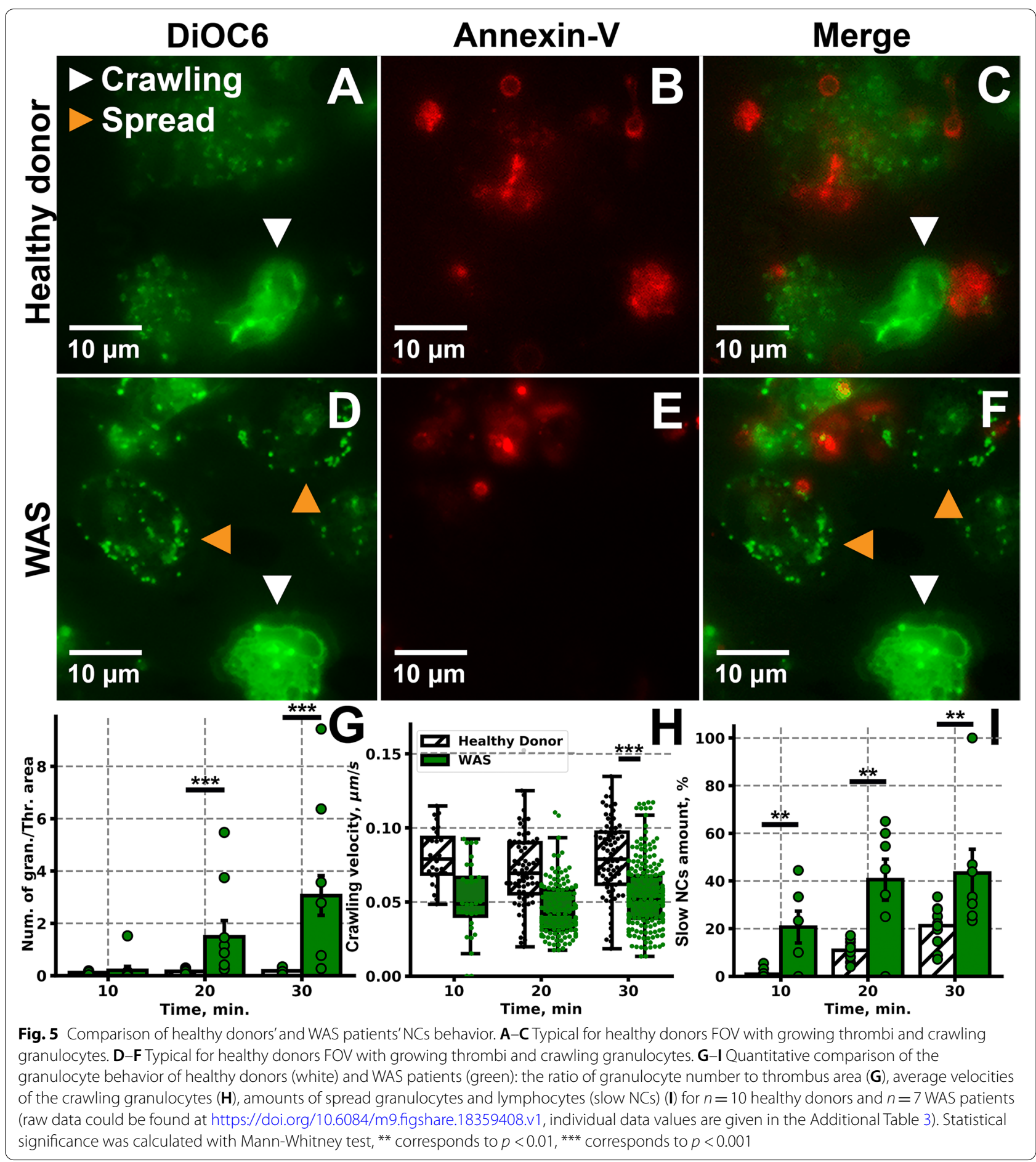

The observed granulocyte incorporation in growing thrombi appeared to be mediated by plasma proteinsfibrinogen and VWF (Fig. 4). For WAS patients, lower crawling velocities and higher relative number of granulocytes were observed (Fig. 5). Thus, the proposed ex vivo experimental setting allows to observe granulocytes activity in near-physiological conditions.

Here, we provide evidence that granulocyte behavior during thrombus formation could be studied ex vivo in parallel-plate flow chambers at wall shear rates less than $200 \mathrm{~s}^{-1}$ and in presence of physiological calcium level 
(Fig. 1, Additional file 1: Fig. S1, Table 1) as well as a substantial amount of fibrinogen and VWF (Fig. 4). We propose hirudin as the most convenient anticoagulant for such studies, because citrate recalcification significantly affects reproducibility of the experiments [34]. Heparin could be an attractive option; however, heparin impact on the immune cell behavior is well established [19, 22, $35,36]$ and was confirmed in this study as well (Additional file 1: Fig. S3, Table 1).

The first quantitative characteristic of granulocytes proposed here is their movement velocity, which appears to be $0.1 \pm 0.02 \mu \mathrm{m} / \mathrm{s}$ on the average, with instant velocities reaching $0.5 \mu \mathrm{m} / \mathrm{s}$ (Table 1 ). These values increased upon priming of granulocytes with various agents (Fig. 2) and decreased in WAS samples (Fig. 5). Previously, C. Jones et al. showed that human neutrophils migrated towards LTB4 with an average velocity of $0.39 \pm 0.09 \mu \mathrm{m} / \mathrm{s}$ [37]. While Jones et al. used a constant gradient of chemoattractant, in our experimental setting, there were several target areas of attraction for granulocytes, which might explain non-monotonous movements of granulocytes in the current study (Fig. 2, Additional files 2 and 3: Video). In another study, M. Weckmann et al. observed the velocity of neutrophil migration on fibronectin in the presence of IL-8, fMLP, and LTB4 [38], where leukocyte velocity appeared to be $0.11 \pm 0.12 \mu \mathrm{m} / \mathrm{s}$, the same as in our study in the presence of the priming reagents (Fig. 2).

The second quantitative characteristic observed here is the percentage of spread granulocytes and attached $\mathrm{T}$ lymphocytes. It has been observed that with the flow of time, granulocytes slow down and spread (Fig. 1, Additional file 6: Video); however, it cannot be claimed that these cells are undergoing NETosis, as it has been demonstrated that NETosis usually occurs later than $30 \mathrm{~min}$ after activation [39]. Analysis of the active CD11b distribution revealed that while active CD11b were present at posterior parts of the crawling cells (Additional file 1: Fig. S4B, Additional file 5: Video), in the spread cells, CD11b was less active and located in the middle part of the cells. This was in agreement with previously published data [40]. It is noteworthy that not only granulocytes participated in the thrombus formation in our setting: a plethora of $\mathrm{T}$ lymphocytes were present as well (Additional file 1: Fig. S4, S5), and thus, their role in thromboinflammation is to be respected as well. Detailed study of the $\mathrm{T}$ lymphocyte activity in the given setting should be the object of further studies.

The third semi-quantitative characteristic is the total number of granulocytes per field of view (Fig. 2). This value depends on the platelet functioning, because it is well-known that platelets activate and attract leukocytes by platelet-leukocyte interactions during thromboinflammation $[1,9,41]$.
Hereby, we assume that our approach can be used for the assessment of platelet-granulocyte interplay in different conditions, including hematological and immunological disorders, such as WAS. We observed impaired granulocyte activity in WAS patients (Fig. 5), consistent with the reduced integrin-dependent degranulation and respiratory burst [42]. The enhanced granulocyte recruitment to the growing thrombi (Fig. 5G) can be the result of the higher proportion of procoagulant platelets in WAS patients $[43,44]$. Supplementation of the WASpatient platelets by healthy donor platelets did not result in a significant increase in the granulocyte velocity that allows to claim that granulocyte motility in WAS patients is caused by WASP deficiency instead of platelet dysfunction (Additional file 1: Fig. S14). It should be noted that additional animal studies could be helpful in order to determine whether direct WASP mutations affect NC crawling in the absence of WASP mutations in platelets.

Several other new findings of platelet-granulocyte interplay were observed in our study. First, we observed procoagulant platelets attached to the moving granulocytes (Fig. 3, Additional file 1: S12). Procoagulant platelets are hyperactivated platelets that underwent mitochondria-dependent necrosis and exposed phosphatidylserine on their surface in the first minutes upon activation [45]. These cells have compromised cytoskeleton what results in their increase in size and loss of most of adhesive integrins [43]. Procoagulant platelets in our conditions attracted granulocytes in the manner common for any tissue debris (Fig. 3), as demonstrated in several studies [46].

Based on our results, we also claim that the mechanism of the granulocyte involvement in thrombus formation is dependent on plasma proteins (fibrinogen and VWF) (Fig. 4A-F). This is in line with findings of Constantinescu-Bercu A. et al. [47] who demonstrated VWF- and platelet integrin $\alpha \mathrm{IIb} \beta 3$-dependent activation of neutrophils and with findings of Ghasemzadeh M. et al. [48], who confirm the essential role of fibrin in intravascular leukocyte trafficking. Additionally, the VWF role in the recruitment of leukocytes during thromboinflammation has been previously established [49].

\section{Conclusions}

In this study, we report the phenomenon of the granulocyte crawling among the growing thrombi ex vivo. We claim that granulocyte characterization can be used for a more in-depth analysis of the mechanisms of immunological and hematologic diseases. Based on our own experimental assays, we propose a scheme of granulocyte participation in thrombus formation: (1) granulocytes attach to growing thrombi in a calcium-dependent manner, (2) granulocyte descent to collagen level is mediated 
by fibrinogen and VWF, (3) descended granulocytes collect annexin-V-positive platelets from the growing thrombi, and (4) granulocytes eventually slow down and arrest (Fig. 4).

\section{Methods}

Aim

The key aim of the study was to establish the experimental setting for the ex vivo observation and characterization of the immune cell participation in the process of venule-thrombus formation by means of fluorescent microscopy.

\section{Materials}

The sources of the materials were as follows: Annexin V-Alexa Fluor 647 (BioLegend, San Diego, CA), DiOC-6, Fucoidan from Fucus vesiculosis, HEPES, bovine serum albumin, lipopolysaccharides from E. coli O111:B4, human fibrinogen, Hoechst-33342 (Sigma-Aldrich, St Louis, MO); CD11b-FITC, CD18-APC, CD66aceAlexa647, CD66b-PE, CD2-APC (Sony Biotechnology, San Jose, CA), fibrillar collagen type I (Chrono-Log Corporation; Havertown; USA); human von Willebrand factor (VWF) was a kind gift of Prof. Pierre Mangin (NSERM, Etablissement Français du Sang-Grand Est, UMR_S1255, Fédération de Médecine Translationnelle de Strasbourg, Université de Strasbourg, France). The HL-60 cell line (promyelocytic leukemia) was used as a source of myeloperoxidase (MPO). MPO was isolated, as described in [50]. LAL-test was performed on human fibrinogen and VWF using LAL-kit Lonza (QCL-1000) in order to ensure absence of the endotoxin contamination (Additional file 1: Tables S1, S2).

\section{Blood collection and handling}

Blood collection was performed under the protocol approved by the free CTP PCP RAS Ethical Committee (protocol \#1 from 12.01.2018), and written informed consents were obtained from all donors and patients. Blood was collected from healthy adult volunteers ( $n=35$, men and women 18-35years old) into Vacuette $\odot$ sodium citrate $(3.8 \% \mathrm{v} / \mathrm{v})$ or lithium heparin (18 I.U./ $\mathrm{ml}$ blood) or Sarstedt-Monovette $\odot$ hirudin (525 ATU/ $\mathrm{ml}$ blood) vacuum tubes. Experiments were performed within $3 \mathrm{~h}$ after blood collection. For the assays involving Wiskott-Aldrich syndrome patients, blood was collected from healthy pediatric donors $(n=12)$ or from patients with Wiskott-Aldrich syndrome $(n=10)$ into SarstedtMonovette hirudin (525 ATU/ml blood) tubes.

For the experiments with "hybrid" and "plasma-free" settings, blood samples were purified from plasma proteins by 3 sequential centrifugations of citrated whole blood for $10 \mathrm{~min}$ by $1000 \mathrm{~g}$ with supplementation of the plasma by Tyrode's calcium-free buffer. Final supplementation was performed by Tyrode's calcium buffer. For artificial thrombocytopenia studies, whole citrated blood of healthy donors was centrifuged at $100 \mathrm{~g}$ for $8 \mathrm{~min}$. PRP was collected above the buffy coat and centrifuged at 1000 for $10 \mathrm{~min}$. The resultant supernatant was then collected and returned to the whole blood. For control experiments, PRP was returned to the blood without additional centrifugation. Platelet count was monitored using Drew D3 cell counter (Drew Scientific, USA). For WAS-hybrid patient studies patients' samples were depleted from platelets in the same manner. Platelets of healthy donors were washed by sequential centrifugations as described earlier [45] (100 $\mathrm{g} 8 \mathrm{~min}$ for PRP and then two centrifugations for $10 \mathrm{~min}$ at $1000 \mathrm{~g}$ with resuspension in Tyrode's buffer) and concentrated 10 times above initial. Healthy donor platelets were then added to the WAS patient platelet-depleted blood. Prior to the experiments, the sample was recalcified to achieve free calcium concentration of $2.5 \mathrm{mM}$.

\section{Fluorescent microscopy}

Parallel-plate flow chambers were described previously [30]. Channel parameters were as follows: $0.2 \times 18 \times 0.206 \mathrm{~mm}$. Glass coverslips were coated with fibrillar collagen type I $(0.2 \mathrm{mg} / \mathrm{ml})$ for $1 \mathrm{~h} 30 \mathrm{~min}$ at $37{ }^{\circ} \mathrm{C}$, washed with distilled water and then inserted into the flow chambers. Blood was perfused through the parallel-plate chambers over collagen-coated $(0.2 \mathrm{mg} / \mathrm{ml})$ surface with wall shear rates $100 \mathrm{~s}^{-1}$ [51]. Thrombus growth and leukocyte crawling were observed in DIC/epifluorescence modes with an inverted Nikon Eclipse Ti-E microscope (100x/1.49 NA TIRF oil objective).

\section{Data analysis}

Nikon NIS-Elements software was used for microscope image acquisition; ImageJ (http://imagej.net/ImageJ) was used for image processing. ImageJ manual tracking plugin was used for manual granulocyte tracking, and the Coloc2 plugin for fluorescence colocalization analysis plugin was utilized. For automated cell tracking, particle tracking algorithm described in [52] was utilized. The algorithm was based on Python trackpy v 0.4.2 library. First, particle tracking was performed, and then the tracks belonging to leukocytes were selected manually. The platelet thrombus area was calculated as the percentage of the screen covered by platelet thrombi upon the subtraction of the area of crawling cells. Tracking Code listing and program operation examples can be found in the data availability statement below. 


\section{Statistics}

All experiments were performed at least in triplicate with platelets from different donors. Statistical analysis was performed using Python 3.6; all statistical details are provided in the figure legends.

\section{Abbreviations}

MPO: Myeloperoxidase; LPS: Lipopolysaccharides from E. coli O111:B4; WWF: von Willebrand factor; PS: Phosphatidylserine; GP: Glycoprotein; CD: Cluster of differentiation; FOV: Field of view; WAS: Wiskott-Aldrich syndrome; NCs: Nuclear cells.

\section{Supplementary Information}

The online version contains supplementary material available at https://doi. org/10.1186/s12915-022-01238-X.

Additional file 1: Figure S1. Comparison of the anti-coagulant impact on NC incorporation to the growing thrombi. Figure S2. Instant NC velocities and averaged velocities. Figure S3. Comparison between heparin and hirudin impact on NCs. Figure S4. Classification of the NC cells among the thrombi. Figure S5. Analysis of the CD11b/CD18 distribution in granulocytes and MNCs. Figure S6. The number of NCs per FOV increased gradually up to the 20th minute of the experiment. Figure S7. Blood cell composition changes after perfusion through flow chamber with fibrillar collagen or BSA. Figure S8. Impact of platelet depletion on granulocyte crawling velocity. Figure S9. Fucoidan impact on platelet P-selectin exposure in growing thrombi. Figure $\mathbf{S 1 0}$. Granulocyte movement around thrombi. Figure S11. Thrombus area and percentage of highly activated granulocytes in the presence of leukocyte activators and in patients with WAS. Figure S12. Crawling granulocytes bear Annexin-V positive platelets. Figure S13. Annexin- $V$ and CD66b staining. Figure S14. Assay of the granulocyte crawling in the blood of WAS patients in the presence of healthy donor platelets. Table S1. LAL-test results for endotoxin characterization in the protein samples. Table S2. Calibration of the LAL-test system.

Additional file 2. Video: Blood anticoagulant impact on NC behavior and thrombus growth. Comparison of the typical Hoechst 33342 and DiOC6 stained NC behavior alongside thrombus growth in the EDTA, sodium citrate, sodium citrate + calcium, heparin, and hirudin anticoagulation.

Additional file 3. Video: Types of NC motion. Typical rolling, crawling, and slow NCs were stained with Hoechst 33342 (magenta) and DiOC6 (green).

Additional file 4. Video: Lymphocyte identification. Videos show that slow NCs with single nuclei (stained by Hoechst 33342 - magenta and DiOC6 - green) are CD2 positive (red) and are thus T-lymphocytes.

Additional file 5. Video: Granulocyte crawling. Crawling cells with polymorphonuclear structure (Hoechst 33342 - magenta) are CD11b (blue) and CD66ace (red) positive. Thus, in the given setting, the observed crawling cells are granulocytes.

Additional file 6. Video: Changes in DiOC6 distribution upon granulocyte activation. Videos of the granulocyte (DiOC6 stained - green) crawling, slowing down and subsequent spreading among the growing thrombi.

Additional file 7. Video: Granulocytes bear Annexin-V+ platelets. Videos of Hoechst 33342 (magenta) labeled granulocytes, which tear away from platelet thrombi (CD61 - blue) procoagulant Annexin-V (red) positive platelets.

Additional file 8. Video: Plasma protein impact on NC attraction to thrombi. Typical timelapse and Z-stack videos of granulocyte behavior upon blood plasma depletion in the absence or the presence of WWF and fibrinogen, accompanied by the standard whole blood experiments.

Additional file 9: Additional Table 3. Individual data values for Figs. 1, 2, 3,5, S3, S6, S7, S8, 59, S11, S14.

\section{Acknowledgements}

We thank Miss. A.E. Boldova and Miss A.A. Petlenko for their assistance during the microscopy data collection.

\section{Authors' contributions}

D.S.M. performed the fluorescent microscopy experiments, analyzed the data, wrote the text, and edited the paper; A.A.M. performed the fluorescent microscopy experiments, analyzed the data, wrote the text, and edited the paper; S.I.O. performed the confocal microscopy experiments; J-J.K. performed the experiments and developed the software for the automated data analysis; A.V.S. isolated the MPO and edited the paper; E.V.S. and I.V.G. analyzed the data and edited the paper; A.Y.S. and A.L.K. managed the patients with WAS and edited the paper; M.A.P. planned the research and edited the paper; A.N.S. supervised the project, planned the research, analyzed the data, performed experiments, and edited the paper. The authors declare that they have no conflict of interest. All authors read and approved the final manuscript.

\section{Funding}

The project was supported by a grant from the endowment foundation "Science for Children" (Moscow, RF), by the Russian Foundation for Basic Research Grants 17-00-00138 and 21-51-10005 (together with the Royal Society of London (RS)); the low-resolution experiments (Additional file 1: Fig. S8, S12, S14) were designed and supported by Russian Science Foundation grant 20-45-01014.

\section{Availability of data and materials}

All data generated or analyzed during this study are included in this published article, its supplementary information files, and publicly available repositories. Python code for the calculation of the crawling granulocyte velocity is uploaded to GitHub repository (minimal: python 3.4; platform independent; requirements - trackpy and anaconda packages for python 3.4 and above): https://github.com/CTPSignalingLab/granulocyte_crawling.git. Raw microscopy data could be found at https://figshare.com/projects/Granulocyte_invol vement_in_thrombus_formation/130217.

\section{Declarations}

\section{Ethics approval and consent to participate}

The research protocols of the given study were approved by the free CTP PCP RAS Ethical Committee (protocol \#1 from 12.01.2018). Written informed consents were obtained from all donors and patients.

\section{Consent for publication}

All authors have read the manuscript and approve of its publication.

\section{Competing interests}

The authors declare that they have no competing interests.

\section{Author details}

${ }^{1}$ Pirogov Russian National Research Medical University, Ostrovityanova Str., 1, Moscow 117997, Russia. ${ }^{2}$ National Medical Research Centre of Pediatric Hematology, Oncology and Immunology named after Dmitry Rogachev, 1 Samory Mashela St, Moscow 117198, Russia. ${ }^{3}$ Center for Theoretical Problems of Physicochemical Pharmacology, Russian Academy of Sciences, 30, Srednyaya Kalitnikovskaya str., Moscow 109029, Russia. ${ }^{4}$ Institute for Experimental Medicine, 12 Acad. Pavlova str., Saint Petersburg 197376, Russia. ${ }^{5}$ Department of Biophysics, Faculty of Physics, Belarusian State University, 220030 Minsk, Belarus. ${ }^{6}$ Faculty of Physics, Lomonosov Moscow State University, 1/2 Leninskie Gory, Moscow 119991, Russia. ${ }^{7}$ Department of Normal Physiology, Sechenov First Moscow State Medical University, 8/2 Trubetskaya St., Moscow 119991, Russia.

Received: 4 March 2021 Accepted: 24 January 2022

Published online: 07 February 2022 


\section{References}

1. Schattner M, Jenne CN, Negrotto S, Ho-Tin-Noe B. Editorial: Platelets and immune responses during thromboinflammation. Front Immunol. 2020;11. https://doi.org/10.3389/fimmu.2020.01079.

2. Darbousset R, Mezouar S, Dignat-George F, Panicot-Dubois L, Dubois C. Involvement of neutrophils in thrombus formation in living mice. Pathol Biol (Paris). 2014;62(1):1-9. https://doi.org/10.1016/j.patbio.2013.11.002

3. Gawaz M, Langer H, May AE. Platelets in inflammation and atherogenesis. J Clin Invest. 2005;115(12):3378-84. https://doi.org/10.1172/JCI27196.

4. Stalker TJ. Platelet activation gradients during thrombus formation. Blood. 2015;126(23):SCI-13. https://doi.org/10.1182/blood.V126.23.SCI-13.SCI-13.

5. Yang $\mathrm{H}$, Lang $\mathrm{S}$, Zhai Z, et al. Fibrinogen is required for maintenance of platelet intracellular and cell-surface P-selectin expression. Blood. 2009;114(2):425-36. https://doi.org/10.1182/blood-2008-03-145821.

6. Rendu F, Brohard-Bohn B. The platelet release reaction: granules' constituents, secretion and functions. Platelets. 2001;12(5):261-73. https://doi. org/10.1080/09537100120068170

7. Kulkarni S, Woollard KJ, Thomas S, Oxley D, Jackson SP. Conversion of platelets from a proaggregatory to a proinflammatory adhesive phenotype: role of PAF in spatially regulating neutrophil adhesion and spreading. Blood. 2007;110(6):1879-86. https://doi.org/10.1182/ blood-2006-08-040980.

8. Ghasemzadeh M, Kaplan ZS, Alwis I, et al. The CXCR1/2 ligand NAP-2 promotes directed intravascular leukocyte migration through platelet thrombi. Blood. 2013;121(22):4555-66. https://doi.org/10.1182/ blood-2012-09-459636.

9. Stegner D, Klaus V, Nieswandt B. Platelets as modulators of cerebral ischemia/reperfusion injury. Front Immunol. 2019;10:2505. https://doi. org/10.3389/fimmu.2019.02505.

10. Perciani CT, Jaoko W, Farah B, et al. aE $\beta 7, a 4 \beta 7$ and $a 4 \beta 1$ integrin contributions to $T$ cell distribution in blood, cervix and rectal tissues: Potential implications for HIV transmission. PLoS One. 2018;13(2):e0192482. https:// doi.org/10.1371/journal.pone.0192482.

11. Lindbom L, Werr J. Integrin-dependent neutrophil migration in extravascular tissue. Semin Immunol. 2002;14(2):115-21. https://doi.org/10.1006/ smim.2001.0348.

12. Arraes SMA, Freitas MS, da Silva SV, et al. Impaired neutrophil chemotaxis in sepsis associates with GRK expression and inhibition of actin assembly and tyrosine phosphorylation. Blood. 2006;108(9):2906-13. https://doi. org/10.1182/blood-2006-05-024638.

13. Perry GS, Spector BD, Schuman LM, et al. The Wiskott-Aldrich syndrome in the United States and Canada (1892-1979). J Pediatr. 1980;97(1):72-8. https://doi.org/10.1016/s0022-3476(80)80133-8.

14. Candotti F. Clinical manifestations and pathophysiological mechanisms of the Wiskott-Aldrich syndrome. J Clin Immunol. 2018;38(1):13-27. https://doi.org/10.1007/s10875-017-0453-z.

15. Mangin PH, Gardiner EE, Nesbitt WS, et al. In vitro flow based systems to study platelet function and thrombus formation: recommendations for standardization: communication from the SSC on Biorheology of the ISTH. J Thromb Haemost. 2020;18(3):748-52. https://doi.org/10.1111/jth.14717.

16. Strobel $\mathrm{L}$, Johswich $\mathrm{KO}$. Anticoagulants impact on innate immune responses and bacterial survival in whole blood models of Neisseria meningitidis infection. Sci Rep. 2018;8(1):10225. https://doi.org/10.1038/ s41598-018-28583-8.

17. Grigorieva DV, Gorudko IV, Kostevich VA, et al. Exocytosis of myeloperoxidase from activated neutrophils in the presence of heparin. Biomed Khim. 2018:64(1):16-22. https://doi.org/10.18097/PBMC20186401016.

18. Khandelwal S, Ravi J, Rauova L, et al. Polyreactive IgM initiates complement activation by PF4/heparin complexes through the classical pathway. Blood. 2018;132(23):2431-40. https://doi.org/10.1182/ blood-2018-03-834598.

19. Gorudko IV, Grigorieva DV, Sokolov AV, et al. Neutrophil activation in response to monomeric myeloperoxidase. Biochem Cell Biol. 2018:96(5):592-601. https://doi.org/10.1139/bcb-2017-0290.

20. Reumaux D, de Boer M, Meijer AB, Duthilleul P, Roos D. Expression of myeloperoxidase (MPO) by neutrophils is necessary for their activation by anti-neutrophil cytoplasm autoantibodies (ANCA) against MPO. J Leukoc Biol. 2003;73(6):841-9. https://doi.org/10.1189/jlb.1102567.

21. Lau D, Mollnau H, Eiserich JP, et al. Myeloperoxidase mediates neutrophil activation by association with CD11b/CD18 integrins. Proc Natl Acad Sci U S A. 2005;102(2):431-6. https://doi.org/10.1073/pnas.0405193102.
22. Grigorieva DV, Gorudko IV, Sokolov AV, et al. Myeloperoxidase stimulates neutrophil degranulation. Bull Exp Biol Med. 2016;161(4):495-500. https:// doi.org/10.1007/s10517-016-3446-7.

23. Johansson MW, Patarroyo M, Oberg F, Siegbahn A, Nilsson K. Myeloperoxidase mediates cell adhesion via the alpha M beta 2 integrin (Mac-1, CD11b/CD18). J Cell Sci. 1997;110(Pt 9):1133-9.

24. Klinke A, Nussbaum C, Kubala L, et al. Myeloperoxidase attracts neutrophils by physical forces. Blood. 2011;117(4):1350-8. https://doi.org/10. 1182/blood-2010-05-284513.

25. Martyanov AA, Balabin FA, Dunster JL, Panteleev MA, Gibbins JM, Sveshnikova AN. Control of platelet CLEC-2-mediated activation by receptor clustering and tyrosine kinase signalling. Biophys J. 2020;0(0). https://doi. org/10.1016/j.bpj.2020.04.023.

26. Jin JO, Yu Q. Fucoidan delays apoptosis and induces pro-inflammatory cytokine production in human neutrophils. Int J Biol Macromol. 2015;73:65-71. https://doi.org/10.1016/j.ijbiomac.2014.10.059.

27. Bylund J, Karlsson A, Boulay F, Dahlgren C. Lipopolysaccharide-induced granule mobilization and priming of the neutrophil response to Helicobacter pylori peptide $\mathrm{Hp}(2-20)$, which activates formyl peptide receptorlike 1. Infect Immun. 2002;70(6):2908-14. https://doi.org/10.1128/iai.70.6. 2908-2914.2002.

28. Martyanov AA, Maiorov AS, Filkova AA, et al. Effects of bacterial lipopolysaccharides on platelet function: inhibition of weak platelet activation. Sc Rep. 2020;10(1):12296. https://doi.org/10.1038/s41598-020-69173-x.

29. Nagata S, Hanayama R, Kawane K. Autoimmunity and the clearance of dead cells. Cell. 2010;140(5):619-30. https://doi.org/10.1016/j.cell.2010.02. 014.

30. Nechipurenko DY, Receveur N, Yakimenko AO, et al. Clot contraction drives the translocation of procoagulant platelets to thrombus surface. Arterioscler Thromb Vasc Biol. 2019;39(1):37-47. https://doi.org/10.1161/ ATVBAHA.118.311390.

31. French AP, Mills S, Swarup R, Bennett MJ, Pridmore TP. Colocalization of fluorescent markers in confocal microscope images of plant cells. Nat Protoc. 2008;3(4):619-28. https://doi.org/10.1038/nprot.2008.31.

32. Rivers $E$, Thrasher AJ. Wiskott-Aldrich syndrome protein: emerging mechanisms in immunity. Eur J Immunol. 2017;47(11):1857-66. https:// doi.org/10.1002/eji.201646715.

33. Martyanov AA, Morozova DS, Sorokina MA, et al. Heterogeneity of integrin allb $\beta 3$ function in pediatric immune thrombocytopenia revealed by continuous flow cytometry analysis. Int J Mol Sci. 2020;21(9):3035. https:// doi.org/10.3390/ijms21093035.

34. Pretorius E, Oberholzer HM, van der Spuy WJ, Franz RC. Comparing techniques: the use of recalcified plasma in comparison with citrated plasma alone and in combination with thrombin in ultrastructural studies. Hematology. 2011;16(6):337-40. https://doi.org/10.1179/102453311X 13127324303515

35. Léculier C, Couprie N, Adeleine P, Leitienne P, Francina A, Richard M. The effects of high molecular weight- and low molecular weight-heparins on superoxide ion production and degranulation by human polymorphonuclear leukocytes. Thromb Res. 1993;69(6):519-31. https://doi.org/10.1016/ 0049-3848(93)90056-t.

36. Videm V. Heparin in clinical doses "primes" granulocytes to subsequent activation as measured by myeloperoxidase release. Scand J Immunol. 1996;43(4):385-90. https://doi.org/10.1046/j.1365-3083.1996.d01-57.x.

37. Jones CN, Hoang AN, Martel JM, et al. Microfluidic assay for precise measurements of mouse, rat, and human neutrophil chemotaxis in whole-blood droplets. J Leukoc Biol. 2016;100(1):241-7. https://doi.org/ 10.1189/jlb.5TA0715-310RR

38. Weckmann M, Becker T, Nissen G, Pech M, Kopp MV. SiMA: a simplified migration assay for analyzing neutrophil migration. Cytometry A. 2017;91(7):675-85. https://doi.org/10.1002/cyto.a.23114.

39. Boeltz S, Amini P, Anders HJ, et al. To NET or not to NET: current opinions and state of the science regarding the formation of neutrophil extracelIular traps. Cell Death Differ. 2019;26(3):395-408. https://doi.org/10.1038/ s41418-018-0261-x

40. Ley K. Integration of inflammatory signals by rolling neutrophils. Immunol Rev. 2002;186(1):8-18. https://doi.org/10.1034/j.1600-065X.2002. 18602.x.

41. Nording HM, Seizer P, Langer HF. Platelets in inflammation and atherogenesis. Front Immunol. 2015;6. https://doi.org/10.3389/fimmu.2015. 00098. 
42. Altman LC, Snyderman R, Blaese RM. Abnormalities of chemotactic lymphokine synthesis and mononuclear leukocyte chemotaxis in WiskottAldrich syndrome. J Clin Invest. 1974;54(2):486-93. https://doi.org/10. 1172/JCl107784.

43. Obydennyi Sl, Artemenko EO, Sveshnikova AN, et al. Mechanisms of increased mitochondria-dependent necrosis in Wiskott-Aldrich syndrome platelets. Haematologica. 2020;105(4):1095-106. https://doi.org/10.3324/ haematol.2018.214460.

44. Shcherbina A, Rosen FS, Remold-O'Donnell E. Pathological events in platelets of Wiskott-Aldrich syndrome patients. Br J Haematol. 1999;106(4):875-83. https://doi.org/10.1046/j.1365-2141.1999.01637.x.

45. Sveshnikova AN, Balatskiy AV, Demianova AS, et al. Systems biology insights into the meaning of the platelet's dual-receptor thrombin signaling. J Thromb Haemost. 2016;14(10):2045-57. https://doi.org/10.1111/jth. 13442.

46. Yuan Y, Alwis I, Wu MCL, et al. Neutrophil macroaggregates promote widespread pulmonary thrombosis after gut ischemia. Sci Transl Med. 2017;9(409):eaam5861. https://doi.org/10.1126/scitransImed.aam5861.

47. Constantinescu-Bercu A, Grassi L, Frontini M, Salles-Crawley II, Woollard K, Crawley JT. Activated allb $\beta 3$ on platelets mediates flow-dependent NETosis via SLC44A2. Elife. 2020;9. https://doi.org/10.7554/eLife.53353.

48. Kaplan ZS, Zarpellon A, Alwis I, et al. Thrombin-dependent intravascular leukocyte trafficking regulated by fibrin and the platelet receptors GPIb and PAR4. Nat Commun. 2015;6:7835. https://doi.org/10.1038/ncomm s8835.

49. Denorme F, Vanhoorelbeke K, De Meyer SF. von Willebrand factor and platelet glycoprotein lb: a thromboinflammatory axis in stroke. Front Immunol. 2019;10:2884. https://doi.org/10.3389/fimmu.2019.02884.

50. Sokolov AV, Acquasaliente L, Kostevich VA, et al. Thrombin inhibits the anti-myeloperoxidase and ferroxidase functions of ceruloplasmin: relevance in rheumatoid arthritis. Free Radic Biol Med. 2015;86:279-94. https://doi.org/10.1016/j.freeradbiomed.2015.05.016.

51. Van Kruchten R, Cosemans JMEM, Heemskerk JWM. Measurement of whole blood thrombus formation using parallel-plate flow chambers - a practical guide. Platelets. 2012;23(3):229-42. https://doi.org/10.3109/ 09537104.2011.630848.

52. Crocker JC, Hoffman BD. Multiple-particle tracking and two-point microrheology in cells. Methods Cell Biol. 2007;83:141-78. https://doi.org/10. 1016/S0091-679X(07)83007-X

\section{Publisher's Note}

Springer Nature remains neutral with regard to jurisdictional claims in published maps and institutional affiliations.

- fast, convenient online submission

- thorough peer review by experienced researchers in your field

- rapid publication on acceptance

- support for research data, including large and complex data types

- gold Open Access which fosters wider collaboration and increased citations

- maximum visibility for your research: over 100M website views per year

At BMC, research is always in progress.

Learn more biomedcentral.com/submissions 